MATHEMATICS OF COMPUTATION

Volume 70, Number 236, Pages 1607-1616

S 0025-5718(01)01305-9

Article electronically published on March 7, 2001

\title{
LOCALIZATION OF THE FIRST ZERO OF THE DEDEKIND ZETA FUNCTION
}

\author{
SAMI OMAR
}

\begin{abstract}
Using Weil's explicit formula, we propose a method to compute low zeros of the Dedekind zeta function. As an application of this method, we compute the first zero of the Dedekind zeta function associated to totally complex fields of degree less than or equal to 30 having the smallest known discriminant.
\end{abstract}

\section{INTRODUCTION}

Let $K$ be a number field of degree $n$, of signature $\left(r_{1}, r_{2}\right)$ and of discriminant $d_{K}$. The Dedekind zeta function of $K$ is denoted by $\zeta_{K}(s)$. Currently there are no efficient methods to compute high zeros of Dedekind zeta functions of general non-abelian fields. For low zeros there is a method of Friedman $[\mathrm{F}$ implemented by Tollis [T], but computations are restricted to number fields of small degree $(n \leq 6)$. In this paper we show how to compute low zeros of the Dedekind zeta function by using Weil's explicit formula and its derivative. Actually, we compute the first zero by reversing the known Serre-Odlyzko construction to bounding discriminants when the field's discriminant is known. This method requires one to compute norms of prime ideals. For that purpose we use efficient algorithms to decompose the minimal polynomial modulo rational primes. As an application of this method, we estimate the first zero of the Dedekind zeta function of totally complex fields of degree less than 30 having the smallest known discriminant CDO.

The functions $F(x)$ used in our computations are Serre's, which in fact give discriminant bounds less good than Odlyzko's but have the interesting property that their Fourier transforms $\hat{F}(x)$ decrease very rapidly and monotonically as $x$ tends to $+\infty$. Therefore the sum running over the non-trivial zeros in Weil's explicit formula is concentrated on the very low zeros of $\zeta_{K}(s)$. We look then to find out how to separate these zeros and estimate the first one with a given precision.

\section{WeIL'S IDENTITY}

Let $F$ be a real function of a real variable which we can suppose even and verifying the following conditions (A) and (B) $[\mathrm{L}$ :

(A) $F$ is continuous and continuously differentiable everywhere except at a finite number of points $a_{i}$, where $F(x)$ and $F^{\prime}(x)$ have only a discontinuity of first kind, such that $F\left(a_{i}\right)=\frac{1}{2}\left(F\left(a_{i}+0\right)+F\left(a_{i}-0\right)\right)$.

Received by the editor June 4, 1999 and, in revised form, February 1, 2000.

2000 Mathematics Subject Classification. Primary 11R42.

Key words and phrases. Dedekind zeta function, zeros, discriminants. 
(B) There is a number $b>0$ such that $F(x)$ and $F^{\prime}(x)$ are $O\left(e^{-\left(\frac{1}{2}+b\right)|x|}\right)$ as $|x| \rightarrow \infty$.

Then the Mellin transform of $F$,

$$
\Phi(s)=\int_{-\infty}^{+\infty} F(x) e^{\left(s-\frac{1}{2}\right) x} d x,
$$

is holomorphic in every strip $-a \leq \sigma \leq 1+a$, where $0<a<b, a<1$, and we have the result established by Weil [Pt1, $\mathrm{Pt2}$ :

Theorem 1 (Weil). Let $F$ satisfy the conditions $(A)$ and $(B)$ above. Then the sum $\sum \Phi(\rho)$ running over the non-trivial zeros $\rho=\beta+i \gamma$ of $\zeta_{K}(s)$ with $|\gamma|<T$ tends to a limit as $T$ tends to infinity, and this limit is given by the formula

$$
\begin{aligned}
\sum_{\rho} \Phi(\rho) & =\Phi(0)+\Phi(1)-2 \sum_{\mathfrak{p}, m} \frac{\ln (N(\mathfrak{p}))}{N(\mathfrak{p})^{\frac{m}{2}}} F(m \ln (N(\mathfrak{p}))) \\
& +F(0)\left[\ln \left(\left|d_{K}\right|\right)-n \ln (2 \pi)\right]-r_{1} J(F)-n I(F),
\end{aligned}
$$

where

$$
J(F)=\int_{0}^{+\infty} \frac{F(x)}{2 \operatorname{ch}\left(\frac{x}{2}\right)} d x \quad \text { and } \quad I(F)=\int_{0}^{+\infty}\left(\frac{F(x)}{2 \operatorname{sh}\left(\frac{x}{2}\right)}-\frac{e^{-x}}{x}\right) d x .
$$

Consequences. If we consider positive functions depending on a positive parameter $y$ with $F(0)=1$ and for which the Fourier transform is also positive, we get the inequality

$$
\ln \left(\left|d_{K}\right|\right) \geq-\Phi(0)-\Phi(1)+n(\ln (2 \pi)+I(F))+r_{1} J(F) .
$$

In the case where $F$ is Serre's or Odlyzko's function, we determine the optimal value of $y$ which gives the best lower bound for $\ln \left(\left|d_{K}\right|\right)$, obtaining the inequalities of Odlyzko and Serre.

\section{Computation of the first ZERo of the Zeta FunCtion}

Let us consider the identity (1) for the functions $F_{y}(x)=e^{-y x^{2}}$, where $y>0$. The Mellin transform $\Phi_{y}(s)$ of $F_{y}$ is

$$
\Phi_{y}(s)=\sqrt{\frac{\pi}{y}} e^{\frac{1}{4 y}\left(s-\frac{1}{2}\right)^{2}},
$$

and the Fourier transform $\varphi_{y}$ of $F_{y}$ is

$$
\varphi_{y}(t)=\sqrt{\frac{\pi}{y}} e^{-\frac{1}{4 y} t^{2}}
$$

If we assume the Generalized Riemann Hypothesis (GRH) for $\zeta_{K}$, we have $\Phi_{y}(\rho)=$ $\varphi_{y}(t)$, where $\rho=\frac{1}{2}+i t$. For every $k \geq 1$, we denote by $t_{k}$ the positive imaginary part of the $k$ th zero of the Dedekind zeta function. Therefore we have the identity

$$
\begin{aligned}
\sum_{\rho} \Phi_{y}(\rho)=\sqrt{\frac{\pi}{y}}: \sum_{t=\Im m(\rho)} e^{-\frac{t^{2}}{4 y}} & =2 \sqrt{\frac{\pi}{y}} e^{\frac{1}{16 y}}-2 \sum_{\mathfrak{p}, m} \frac{\ln (N(\mathfrak{p}))}{N(\mathfrak{p})^{\frac{m}{2}}} e^{-y(m \ln (N(\mathfrak{p})))^{2}} \\
& +\left[\ln \left(\left|d_{K}\right|\right)-n \ln (2 \pi)\right]-r_{1} J(y)-n I(y),
\end{aligned}
$$

where

$$
J(y)=\int_{0}^{+\infty} \frac{e^{-y x^{2}}}{2 \operatorname{ch}\left(\frac{x}{2}\right)} d x \quad \text { and } \quad I(y)=\int_{0}^{+\infty}\left(\frac{e^{-y x^{2}}}{2 \operatorname{sh}\left(\frac{x}{2}\right)}-\frac{e^{-x}}{x}\right) d x .
$$


In section 4 , we will show how to compute the integrals $J(y), I(y)$, and the sum

$$
v(y)=\sum_{\mathfrak{p}, m} \frac{\ln (N(\mathfrak{p}))}{N(\mathfrak{p})^{\frac{m}{2}}} e^{-y(m \ln (N(\mathfrak{p})))^{2}}
$$

with a given precision. This allows us to compute

$$
S(y)=\sum_{t=\Im m(\rho)} e^{-\frac{t^{2}}{4 y}}
$$

for every $y>0$, as well as its derivative

$$
S^{\prime}(y)=\sum_{t=\Im m(\rho)} \frac{t 2}{4 y^{2}} e^{-\frac{t^{2}}{4 y}} .
$$

One can easily see that $t_{1}>0$ if and only if $S(y)<1$ for some $y>0$. Therefore $S(y)$ can be writen $S(y)=2 \sum_{k=1}^{+\infty} e^{-t_{k}^{2} / 4 y}$, which tends to 0 as $y$ tends to 0 .

Let us start by supposing that there exists $\beta>0$ such that $S(\beta)<1$ (i.e. $t_{1}>0$ ). To compute the first zero of $\zeta_{K}(s)$ on the critical line, we proceed in the following manner:

1. Lower bound for the first zero. We use the following trivial bound:

Lemma 1. For every $y>0$ we have

$$
t_{1}>\sqrt{\max \left(-4 y \ln \left(\frac{S(y)}{2}\right), 0\right)} .
$$

In practice we search for the value of $y$ giving the best lower bound $t_{1 \text { min }}$ of $t_{1}$.

2. Upper bound for the first zero. Let us define

$$
H(y)=4 S^{\prime}(y)-S(y)=2 \sum_{k=1}^{+\infty}\left(\frac{t_{k}^{2}}{y^{2}}-1\right) e^{-\frac{t_{k}^{2}}{4 y}} .
$$

To get an upper bound for $t_{1}$, we use the lemma:

Lemma 2. There exists $y_{0}>0$ such that for every $y \geq y_{0}$ we have $H(y) \leq 0$. It follows from the definition of $H(y)$ that $t_{1}<y_{0}$.

Proof. This is a consequence of the asymptotic behaviour of $S(y)$ and $S^{\prime}(y)$ :

$$
\begin{aligned}
& \lim _{y \rightarrow 0^{+}} S(y)=0^{+}, \\
& \lim _{y \rightarrow 0^{+}} S^{\prime}(y)=0^{+},
\end{aligned}
$$

Therefore $\lim _{y \rightarrow 0^{+}} H(y)=0^{+}$and $\lim _{y \rightarrow+\infty} H(y)=-\infty$, proving lemma 2 .

We take for $t_{1 \max }$ the value of $y$ that is a solution of $H(y)=0$.

3. Improvement of the upper bound and separation of the zeros. Let us consider, for every $\alpha>0$,

$$
M_{\alpha}(z)=2 \sum_{k=1}^{+\infty}\left(\frac{t_{k}^{2}}{z^{16}}-1\right) e^{-\alpha \frac{t_{k}^{2}}{z}}
$$

We have the following lemmas: 
Lemma 3. i) For every $z>0, M_{\alpha}(z)$ can be written in terms of $S$ and $S^{\prime}$ in the form

$$
M_{\alpha}(z)=\frac{1}{4 \alpha^{2} z^{14}} S^{\prime}\left(\frac{z}{4 \alpha}\right)-S\left(\frac{z}{4 \alpha}\right) .
$$

ii) For every value of $\alpha$ there exists a constant $C_{\alpha}>0$ such that $t_{1}<C_{\alpha}$ $\left(C_{\alpha}\right.$ is the solution of $\left.M_{\alpha}\left(C_{\alpha}\right)=0\right)$.

The lemma below gives a lower bound for the second zero of $\zeta_{K}$ as a function of $C_{\alpha}$.

Lemma 4. We have the inequality

$$
t_{2}>\sqrt{\max \left(-4 y \ln \left(\frac{S(y)}{2}-e^{\frac{-C_{\alpha}^{2}}{4 y}}\right), 0\right)} .
$$

This inequality arises by ignoring all but the first two terms in $S(y)$.

In practice, we begin by computing the best constant $C_{\alpha}$ given by lemma 3 ; then we search for the optimal value of $y$ which gives a lower bound of $t_{2}$ greater than $C_{\alpha}$.

4. Improvement of the range for the first zero. An upper bound for $t_{2}$ can be obtained by the determination of the root of the function

$$
H(y)-2\left(\frac{t_{1 \min }^{2}}{y^{2}}-1\right) e^{-\frac{t_{1 \min }^{2}}{4 y}}=0
$$

where $y>t_{1 \max }$.

This upper bound of $t_{2}$ can be used to improve the value of $t_{1 \min }$ given by lemma 1 , but in practice, if we are only interested in slight precision for $t_{1}$ (only one or two decimal digits), the improvement of $t_{1 \min }$ seems to be unnecessary because the desired precision can be obtained by simple improvement of the value of $t_{1 \max }$. This can be done in the following manner.

We define the sequences $\left(b_{n}\right)$ and $\left(c_{n}\right)$ by the recurrent formula

$$
\left\{\begin{array}{l}
b_{0}=t_{1 \max }=C_{\alpha}, \\
c_{0}=t_{2 \min }=\sup _{y>0} \sqrt{\max \left(-4 y \ln \left(\frac{S(y)}{2}-e^{-\frac{C_{\alpha}^{2}}{4 y}}\right), 0\right)}
\end{array}\right.
$$

and, for every integer $n$,

$$
\left\{\begin{array}{l}
b_{n+1}=\inf _{y_{0}>y>0} \sqrt{-4 y \ln \left(\frac{S(y)}{2}-e^{\left.-\frac{c_{n}^{2}}{4 y_{0}}\left(\frac{y_{0}}{y}-1\right) \frac{S\left(y_{0}\right)}{2}\right)}\right.} \\
c_{n+1}=\sup _{y>0} \sqrt{\max \left(-4 y \ln \left(\frac{S(y)}{2}-e^{-\frac{b_{n+1}^{2}}{4 y}}\right), 0\right)} .
\end{array}\right.
$$

We should remark that the formula for $b_{n+1}$ arises by comparing $S(y)$ and $S\left(y_{0}\right)$ term by term and using the fact that, for $0<y<y_{0}$ and $k \geq 2$,

$$
e^{-\frac{c_{n}^{2}}{4 y}+\frac{c_{n}^{2}}{4 y_{0}}} e^{-\frac{t_{k}^{2}}{4 y_{0}}} \geq e^{-\frac{t_{k}^{2}}{4 y}} .
$$

One can prove the lemma below: 
Lemma 5. i) For every integer $n \geq 1$, we have $t_{1} \leq b_{n}$.

ii) For every integer $n \geq 1$, we have $c_{n} \leq t_{2}$.

Now, if we have $t_{1}=0$, that means in practice that $S(y)>1$ for small values of $y$. In this case we don't apply lemma 1 , we just find an upper bound near 0 for $t_{1}$ by combining $S(y)$ and $S^{\prime}(y)$ as in lemma 2 and lemma 3.

The method we have just explained, allowing us to approximate the first zero, can be generalized for the computation of the other zeros $\left(t_{2}, t_{3}, \ldots\right)$.

\section{Computation of $S(y)$}

In this section we give a method to compute the different terms of $S(y)$, i.e., $J(y), I(y)$ and $v(y)$.

4.1. Computation of $J(y)$. Let us write $G_{y}(x)=\frac{e^{-y x^{2}}}{2 \operatorname{ch}\left(\frac{x}{2}\right)}$; then we have:

\section{Lemma 6.}

$$
\lim _{h \rightarrow 0^{+}} \lim _{N \rightarrow+\infty} h\left[\frac{G_{y}(0)}{2}+\sum_{q=1}^{N} G_{y}(q h)\right]=\int_{0}^{+\infty} G_{y}(x) d x .
$$

Proof. We apply Poisson's equality to the function defined by $x \mapsto G_{y}(h x)$, where $h>0$.

In practice we consider two arbitrary sequences $\left(N_{i}\right)$ and $\left(h_{i}\right)$ such that $h_{i} N_{i} \rightarrow$ $+\infty$ and $h_{i} \rightarrow 0$; we will take for example $N_{i} h_{i}^{2}=$ const. When this is the case, the following lemma $\mathrm{DaR}$ shows that the series decreases as $e^{-\operatorname{cons} \sqrt{N}}$.

Lemma 7 (Martensen). Let

$$
E_{h}\left(G_{y}\right)=\int_{0}^{+\infty} G_{y}(x) d x-h\left[\frac{G_{y}(0)}{2}+\sum_{q=1}^{N} G_{y}(q h)\right] .
$$

Then there exists $s_{0}>0$ such that:

$$
\left|E_{h}\left(G_{y}\right)\right| \leq \frac{1}{e^{\frac{2 \pi s_{0}}{h}}-1} \int_{-\infty+i s_{0}}^{+\infty+i s_{0}}\left|G_{y}(z)\right| d z
$$

4.2. Computation of $I(y)$. Let us consider the function $\mathcal{G}_{y}$ defined by

$$
\mathcal{G}_{y}(x)=\frac{1-e^{-y x^{2}}}{2 \operatorname{sh}\left(\frac{x}{2}\right)} .
$$

$I(y)$ can be decomposed as $I(y)=I_{2}-I_{1}(y)$, where

$$
I_{1}(y)=\int_{0}^{+\infty} \mathcal{G}_{y}(x) d x \quad \text { and } \quad I_{2}=\int_{0}^{+\infty}\left(\frac{1}{2 \operatorname{sh}\left(\frac{x}{2}\right)}-\frac{e^{-x}}{x}\right) d x .
$$

The value of $I_{2}$ can be computed by the Gauss formula [GR], which gives $I_{2}=$ $\gamma+2 \ln (2)$. For $I_{1}(y)$ we write

$$
I_{1}(y)=\underbrace{\int_{0}^{1} \mathcal{G}_{y}(x) d x}_{K_{1}(y)}+\underbrace{\int_{1}^{+\infty} \frac{1}{2 \operatorname{sh}\left(\frac{x}{2}\right)} d x}_{K_{2}}-\underbrace{\int_{1}^{+\infty} \frac{e^{-y x^{2}}}{2 \operatorname{sh}\left(\frac{x}{2}\right)} d x}_{K_{3}(y)}
$$


We have $K_{2}=\ln \left(\operatorname{coth} \frac{1}{4}\right)$. To compute $K_{3}(y)$, we apply again lemma 6 , which remains true for the function

$$
G_{y}(x)=\frac{e^{-y(x+1)^{2}}}{2 \operatorname{sh}\left(\frac{x+1}{2}\right)} .
$$

For the computation of $K_{1}(y)$, we use the Clenshaw and Curtis method [DaR] based on the Fourier-Tschebyscheff expansion of $\mathcal{G}_{y}$ on the interval $[-1,1]$.

4.3. Computation of $v(y)$. In practice, we compute

$$
v_{p_{0}}(y)=\sum_{p \leq p_{0}} \sum_{\mathfrak{p} \mid(p)} \ln (N(\mathfrak{p})) \sum_{\substack{m \\ m \ln (N(\mathfrak{p})) \leq c o n s}} \frac{e^{-y(m \ln (N(\mathfrak{p})))^{2}}}{N(\mathfrak{p})^{\frac{m}{2}}},
$$

where cons $=\sqrt{\frac{c \ln (10)}{y}}$. The number field being defined by a polynomial $P(x)$, for every prime number $p$ prime to the index of the field, the decomposition of the ideal $(p)$ into a product of prime ideals of the field is given by the decomposition of $P(x)$ modulo $p[\mathrm{Co}$. In the case where $p$ divides the index, we use a stronger algorithm (see algorithm 6.2.5 in [Co]).

The condition $m \ln (N(\mathfrak{p}))<$ cons means that we don't take into account the terms of the series less than $10^{-c}$. Generally, we take $c=30$, and $p_{0}$ is the greatest prime number less than $10^{7}$ (see section 5 ).

The error term

$$
r_{p_{0}}(y)=v(y)-v_{p_{0}}(y)
$$

is estimated using the following lemmas:

Lemma 8. Let $n$ be $[K: \mathbb{Q}]$. Then

$$
r_{p_{0}}(y) \leq r_{0}(n)+r_{\infty}(n),
$$

where

$$
r_{0}(n)=n^{2} 10^{-c} \sum_{p \leq p_{0}} \sum_{m \ln (p)>\frac{c o n s}{n}} \frac{\ln (p)}{p^{\frac{m}{2}}},
$$

and

$$
r_{\infty}(n)=n^{2} \sum_{p>p_{0}} \sum_{m \geq 1} \ln (p) \frac{e^{-y(m \ln (p))^{2}}}{p^{\frac{m}{2}}} .
$$

Lemma 9 (Estimation of $r_{0}(n)$ ). For every integer $p_{0} \geq 2$ we have

$$
r_{0}(n) \leq 8 \ln (2) n^{2} 10^{-c} e^{-\frac{c o n s}{2 n}} p_{0} .
$$

Proof. We have

$$
r_{0}(n) \leq n^{2} 10^{-c} \sum_{p \leq p_{0}} \frac{\ln (p)}{p^{\frac{g(p)}{2}}\left(p^{\frac{1}{2}}-1\right)},
$$

where $g(p)=E\left(\frac{\text { cons }}{n \ln (p)}\right)$ (here $E(x)$ denotes the floor of $\left.x\right)$. 

by

Thus $r_{0}(n) \leq 4 n^{2} 10^{-c} e^{-\frac{c o n s}{2 n}} \theta\left(p_{0}\right)$, where $\theta$ is the Tschebyscheff function defined

$$
\theta\left(p_{0}\right)=\sum_{p \leq p_{0}} \ln (p)
$$

According to Tschebyscheff's lemma [EM] we have $\theta\left(p_{0}\right) \leq 2 \ln (2) p_{0}$, and the desired result is obtained.

Proposition 1. Let $f$ be a decreasing, continuously differentiable and integrable positive function in $\left[a,+\infty\left[(a \geq 0)\right.\right.$ satisfying $f(x)=o\left(\frac{1}{x}\right)$ and $x f(x) e^{-c \sqrt{\ln (x)}}=$ $o\left(\int_{x}^{+\infty} f(t) d t\right)$ as $|x| \rightarrow \infty$, where $c$ is a positive constant. Then

$$
\sum_{p \geq x} \ln (p) f(p) \sim \int_{x}^{+\infty} f(t) d t .
$$

Proof. Let us consider the function $\theta(x)=\sum_{p \leq x} \ln (p)$. There exists a constant $c>0$ such that $\theta(x)=x+R(x)$, where $R(x)=O\left(x e^{-c \sqrt{\ln (x)}}\right)$ La]. We can write the series $\sum_{p \geq x} \ln (p) f(p)$ as an integral:

$$
\sum_{p \geq x} \ln (p) f(p)=\int_{x}^{+\infty} f(t) d \theta(t)
$$

now by integration by parts, we get

$$
\sum_{p \geq x} \ln (p) f(p)=-f(x) R(x)+\int_{x}^{+\infty} f(t) d t-\int_{x}^{+\infty} f^{\prime}(t) R(t) d t .
$$

It is not difficult to see that $f(x) R(x)=o\left(\int_{x}^{+\infty} f(t) d t\right)$ and

$$
\int_{x}^{+\infty}\left|f^{\prime}(t) R(t)\right| d t=O\left(e^{-c \sqrt{\ln (x)}} \int_{x}^{+\infty}\left|t f^{\prime}(t)\right| d t\right) .
$$

After another integration by parts of this last integral, we find that

$$
\int_{x}^{+\infty}\left|t f^{\prime}(t)\right| d t=x f(x)+\int_{x}^{+\infty} f(t) d t
$$

and we deduce the result of the proposition.

Corollary 1 (Estimation of $\left.r_{\infty}(n)\right)$. We have

$$
r_{\infty}(n) \leq s_{1}(n)+s_{2}(n)+s_{3}(n),
$$

where

$$
\begin{aligned}
& s_{1}(n)=n^{2} e^{-y\left(3 \ln \left(p_{0}\right)\right)^{2}} \sum_{p>p_{0}} \frac{\ln (p)}{p\left(p^{\frac{1}{2}}-1\right)} \\
& \underset{p_{0} \rightarrow \infty}{\sim} 2 n^{2} \frac{e^{-y\left(3 \ln \left(p_{0}\right)\right)^{2}}}{\sqrt{p_{0}}}, \\
& s_{2}(n)=n^{2} \sum_{p>p_{0}} \frac{\ln (p)}{\sqrt{p}} e^{-y \ln (p)^{2}} \\
& \underset{p_{0} \rightarrow \infty}{\sim} \frac{n^{2}}{2 y} \frac{\sqrt{p_{0}} e^{-y \ln \left(p_{0}\right)^{2}}}{\ln \left(p_{0}\right)},
\end{aligned}
$$


and

$$
\begin{aligned}
s_{3}(n) & =n^{2} \sum_{p>p_{0}} \frac{\ln (p)}{p} e^{-y(2 \ln (p))^{2}} \\
& \sim \frac{n^{2}}{8 y} \frac{e^{-4 y \ln \left(p_{0}\right)^{2}}}{\ln \left(p_{0}\right)} .
\end{aligned}
$$

Example. In the case of the totally imaginary field of degree 20 having the smallest known discriminant, the value of $y$ which maximizes $S(y)$ is 0.069 . If we take $p_{0}=4 \cdot 10^{7}$ and $c=30$, the error in the computation of $v(y)$ is bounded by $10^{-4}$.

\section{Application to the computation \\ OF THE FIRST ZERO OF THE DEDEKIND ZETA FUNCTION OF CERTAIN FIELDS OF SMALL DISCRIMINANT}

In the following tables we indicate the value of the first zero $\frac{1}{2}+i t_{1}$ of $\zeta_{K}$, the limit $p_{0}$ of primes taken into consideration in the sum running over the prime ideals of the field, the value $y$ giving the best lower bound for the first zero (cf. lemma 1), and a bound for the error term $r_{p_{0}}(y)$ in the sum $v(y)$ (cf section 4.3).

5.1. Totally real field of small discriminant of degree 7. This field having the smallest discriminant $\left(d_{K}=20134393\right)$ is defined by the polynomial [Ph]:

$$
P(x)=x^{7}+x^{6}-6 x^{5}-5 x^{4}+8 x^{3}+5 x^{2}-2 x-1 ;
$$

and its root discriminant is $14.909 \%$ above the GRH bounds. See Table 1 .

TABLE 1.

\begin{tabular}{|c|c|c|c|}
\hline $\mathbf{t}_{\mathbf{1}}$ & $\mathbf{p}_{\mathbf{0}}$ & $\mathbf{y}$ & $\mathbf{r}_{\mathbf{p}_{\mathbf{0}}}(\mathbf{y})$ \\
\hline 1.81803 & $8 \cdot 10^{7}$ & 0.082 & $10^{-7}$ \\
\hline
\end{tabular}

5.2. Totally imaginary fields of small discriminant of degree less than $\mathbf{3 0}$. We consider here the imaginary complex number fields given in [CDO] of degree less than or equal to 30 having a root discriminant near from Odlyzko's lower bound.

Table 2 shows that the precision of the computations decreases with the degree; for example in degree 24, it was necessary to compute the decomposition for all the primes less than $15 \cdot 10^{7}$ to obtain an error on $t_{1}$ less than $10^{-2}$. In degrees 28 and 30 , the first zero is computed only to $10^{-1}$ but the indicated value is less than the exact value of the first zero. If we don't assume (GRH), we compute $S(y)=\sum \Phi_{y}(\rho)$ for every value $y$ and we obtain the following result:

Lemma 10. The following assertions are equivalent:

i) $S(y)$ is bounded as $y \rightarrow 0$.

ii) $S(y) \rightarrow 0$ as $y \rightarrow 0$.

iii) There are no zeros of the Dedekind zeta function in the triangle $A\left(\frac{1}{2}, 0\right)$, $B(1,0), C\left(1, \frac{1}{2}\right)$.

Remark. Because $S(y)$ is bounded as $y \rightarrow 0$ for all the considered fields, we can deduce the non-existence of a Siegel's zero for these fields. 
TABLE 2 .

\begin{tabular}{|c|c|c|c|c|}
\hline $\mathbf{n}$ & $\mathbf{t}_{\mathbf{1}}$ & $\mathbf{p}_{\mathbf{0}}$ & $\mathbf{y}$ & $\mathbf{r}_{\mathbf{p}_{\mathbf{0}}}(\mathbf{y})$ \\
\hline 2 & 8.03 & $10^{7}$ & 0.4 & $10^{-43}$ \\
\hline 4 & 4.39 & $10^{7}$ & 0.3 & $10^{-31}$ \\
\hline 6 & 3.30 & $10^{7}$ & 0.2 & $10^{-19}$ \\
\hline 8 & 2.75 & $10^{7}$ & 0.11 & $10^{-8}$ \\
\hline 10 & 2.40 & $10^{7}$ & 0.097 & $10^{-6}$ \\
\hline 12 & 2.17 & $10^{7}$ & 0.090 & $10^{-5}$ \\
\hline 14 & 2.10 & $10^{7}$ & 0.085 & $10^{-5}$ \\
\hline 16 & 1.87 & $2 \cdot 10^{7}$ & 0.075 & $10^{-4}$ \\
\hline 18 & 1.76 & $2 \cdot 10^{7}$ & 0.073 & $10^{-4}$ \\
\hline 20 & 1.66 & $4 \cdot 10^{7}$ & 0.069 & $10^{-4}$ \\
\hline 22 & 1.63 & $9 \cdot 10^{7}$ & 0.062 & $10^{-3}$ \\
\hline 24 & 1.54 & $15 \cdot 10^{7}$ & 0.058 & $10^{-3}$ \\
\hline 26 & 1.23 & $6 \cdot 10^{7}$ & 0.057 & $10^{-3}$ \\
\hline 28 & $1.48^{*}$ & $5 \cdot 10^{7}$ & 0.056 & $10^{-2}$ \\
\hline 30 & $1.36^{*}$ & $5 \cdot 10^{7}$ & 0.056 & $10^{-2}$ \\
\hline
\end{tabular}

In conclusion I would like to thank Professor F. Diaz y Diaz for his help in the preparation of this paper, and also to thank M. Balazard for his fruitful suggestions.

\section{REFERENCES}

[CDO] H. Cohen, F. Diaz y Diaz and M. Olivier, A table of totally complex number fields of small discriminants, Algorithmic Number Theory, (Lectures Notes in Computer Science; Springer Verlag, 1998), pp. 381-391. CMP 2000:05

[Co] H. Cohen, A course in computational algebraic number theory, (Graduate Texts in Maths; Springer Verlag, 1993). MR 94i:11105

[DaR] P. Davis and P. Rabinowitz, Methods of numerical integration, (Academic Press, 1975). MR 56:7119

[EM] W. J. Ellison and M. Mendès France, Les nombres premiers, (Hermann, 1975). MR 54:5138

[F] E. Friedman, Hecke's integral formula, Semin. Theor. Nombres Bordeaux (1987-1988), Exp 5, 23 pp. MR 90i:11136

[GR] I. S. Gradshteyn and I. M. Ryzhic, Table of integrals, series and products, (Academic Press, Inc., 1980). MR 81g:33001

[L] S. Lang, Algebraic number theory, (Addision Wesley, 1970). MR 44:181

[La] E. Landau, Vorlesungen über Zahlentheorie, (Chelsea Publishing Company, 1969). MR 40:4076 
[O] A. M. Odlyzko, Bounds for discriminants and related estimates for class numbers, regulators and zeros of zeta functions: A survey of recent results., Semin. Theor. Nombres Bordeaux 2 (1990), 119-141. MR 91i:11154

[Ph] M. Pohst, The minimum discriminant of seventh degree totally real algebraic number fields, Number Theory and Algebra (Academic Press) (1977), 235-240. MR 57:5952

[Pt1] G. Poitou, Minorations de discriminants, Séminaire Bourbaki, 28e année, 1975/76, n 479.

[Pt2] G. Poitou, Sur les petits discriminants, Séminaire Delange-Pisot-Poitou, 18e année, 1976/77, n 6 = Lecture Notes in Math. 567 (1977), 136-153. MR 55:7995

[T] E. Tollis, Zeros of Dedekind zeta functions in the critical strip, Math. Comp. 66 (1997), 1295-1321. MR 98d:11140

Laboratoire d'Algorithmique Arithmétique, Université Bordeaux I, 351 Cours de la Libération, F-33405 Talence Cedex France

E-mail address: omar@math.u-bordeaux.fr 